\title{
Sexual and reproductive health as a research topic, which acceptability among Moroccan youth?
}

\author{
Jamila El Hassouni ${ }^{*}, 1,2$, Nabila Rouahi $^{1}$, and Meftaha Senhaji ${ }^{2}$ \\ ${ }^{1}$ Higher Institute of the Nursing Professions and Health Techniques, Tetouan, Morocco \\ ${ }^{2}$ Faculty of Sciences, Abdelmalek Essaâdi University, Tetouan, Morocco
}

\begin{abstract}
As a previous step of a quantitative study about sexual and reproductive health (SRH) among young Moroccan adults, and because of the sensitivity of this field, we aimed to examine its feasibility and acceptability through a qualitative approach. We conducted four focus groups composed of 26 participants from both sexes and aged from 18 to 23 years old, studying at the Higher Institute of Nursing Professions and Health Techniques of Tetouan. We carried out the coding and categorizing the verbatim transcripts using the QRS Nvivo v.10.0. The results show that the majority of participants do not know the concept of SRH except for some components such as HIV/AIDS and family planning and that the Internet is their leading source of information. They also show the influence of social norms and religion on sexual practices among female participants, whereas sexuality was the main concern of male participants. Therefore, sex and education seem to be the best way to solve SRH related problems. In fact, participants insisted on its integration into the basic school curriculum. They also expressed their need for an official website about SRH managed by experts as well as a health service specialized in SRH, both specific to adolescents and youth. We conclude that a quantitative study on this topic is feasible and acceptable in our context.
\end{abstract}

\section{Introduction}

Young people are essential to families, communities, and society. Nevertheless, too many young people still go from childhood to adulthood without having had accurate, complete, or non-judgmental information about their sexual health, which affects their physical, social, and emotional development and makes them more vulnerable to exploitation and other harmful consequences [1]. The International Conference on Population and Development (ICPD) Program of Action, held in Cairo in 1994, recognized that everyone has the right to achieve the highest standard of sexual and reproductive health. ICPD also includes their right to make decisions about reproduction without discrimination, coercion, and violence, as expressed in human rights documents [2]. However, according to the UNAIDS Report on the Global AIDS Epidemic (2008), only 40\% of young people aged between 15 and 24 years old have specific knowledge about HIV and its transmission [3]. Culture and beliefs also influence young people's SRH. In many countries, parents cannot discuss sensitive topics with their children due to the 'culture of shame', which prevents adults, and children from talking about their bodies or their sexual activity, and makes it difficult to ask for help in the aftermath of violence and sexual assault [4].

Moreover, despite the efforts made to meet the needs of adolescents and youth better, their SRH situation in Morocco remains very worrying. The National Multiple Indicator and Youth Health Survey (ENIMSJ, 2006-2007) revealed that the 15-24 age group is vulnerable [5]:

$-12 \%$ of girls had an unwanted pregnancy resulting from unprotected sex;

$-8 \%$ of young people aged $15-24$ years have AIDS;

$-15 \%$ of boys and $19 \%$ of girls said they did not have enough information about the signs of puberty;

$-15 \%$ of young people do not know any method of contraception, and $40 \%$ do not use any of these methods Furthermore, one in five young people does not know about HIV/AIDS and nine out of ten young people do not know about other sexually transmitted infections STIs [6]. Based on this alarming situation, and following our knowledge that no study has been dealing with the subject of sexuality and reproduction among young university students in the city of Tetouan, we decided to determine the situation among this population. Given the sensitivity of the topic of SRH, we decided to conduct preliminary exploratory research to assess the feasibility and acceptability of addressing this topic among youth first before moving to a large-scale study.

\footnotetext{
"Corresponding author: j.elhassouni@gmail.com
} 


\section{Methods}

In this study, we adopted a qualitative approach for two reasons: first, to explore the sensitive nature of our research topic in terms of the acceptability of the process to the concept of SRH by young people at the Higher Institute of the Nursing Professions and Health Techniques (ISPITS) of Tetouan, as well as their knowledge and future needs regarding sexuality and procreation; and second, as a preliminary to quantitative research to explore new areas, concepts, and before behaviours beginning to measure them [7].

Indeed, in order to gather information about the overall knowledge and experiences of young people in matters of sexuality, SRH, and access to services [8], we conducted four homogeneous focus groups (FGs) (two female FGs and two male FGs). We collected all data in January and February 2021.

\subsection{Participants and settings}

We held four FGs composed of 5-10 participants, with a total of 26 participants, including 15 males (M) and 11 females (F). The age range of participants was 18 to 23 years old: a majority (75\%) was between 18 and 19 years old. $56 \%$ live in Tetouan and $44 \%$, in its region (Chefchaouen and Ouazzane). All participants follow the courses of the first semester of the first year in the ISPITS of Tetouan. We excluded students who were at least in the second semester because they have learnt about contraception and IST in their training lessons. Table 1 summarizes the details about participants.

Table 1: Characteristics of focus group participants $(\mathrm{N}=26)$

\begin{tabular}{cccccccc} 
& \multicolumn{3}{c}{ Age } & \multicolumn{2}{c}{ Habitat } & \multicolumn{2}{c}{ Sex } \\
\cline { 2 - 7 } & $18-19$ & $20-21$ & $22-23$ & T & TR & F & M \\
\hline \multirow{2}{*}{$\mathrm{N} \%$} & 19 & 5 & 2 & 18 & 8 & 11 & 15 \\
& $75 \%$ & $20 \%$ & $5 \%$ & $56 \%$ & $44 \%$ & $39 \%$ & $61 \%$ \\
\hline
\end{tabular}

T: Tetouan; TR: Tetouan region; F:females; M: males

FGs lasting from 90 to 150 minutes were conducted by the authors and trained facilitators from the last year of the license cycle (midwife's students with female groups and nursing students with male groups). They used solely the mother tongue to facilitate participants' expression. Active listening and reformulations were very beneficial to encourage participants to express themselves better and to ensure that the information was correctly understood [9]. We collected data using an interview guide developed according to relevant literature, and recorded the audios.

\subsection{Data analysis}

We transcribed verbatim the audio files from the FGs and translated them into French. The analysis approach that we followed is the thematic analysis [10]. We carried out the entry of the transcripts as well as the coding and analysis of the content by using QSR NVivo v.10.0 software.

\subsection{Ethical consideration}

We made all provisions to preserve the rights of the study participants:

1) The right to anonymity and confidentiality;

2) The right to self-determination: they voluntarily chose whether to collaborate or not in the study, and had the right to withdraw from the research at any time without having to justify it while being assured that their decision to participate or not would have no effect on their studies;

3) Free and informed consent: free consent to participate in the study was taken after explaining the purpose of the research.

\section{Results}

The FGs data analysis allowed us to emerge five main themes: (1) the feasibility of approaching the field of SRH, (2) the Reproductive aspect, (3) the Aspects of sexual health, (4) care delivery and information sources, and (5) recommendations. We will present the results concerning the first theme: the feasibility of approaching the field of SRH with youth.

All participants expressed openness to the topic of SRH despite the notion of taboo surrounding reproduction and sexuality. Indeed, they have difficulties in broaching this type of discussion with parents or relatives:

“... a taboo subject (shameful) in our society and no one wants to talk about this subject when it is a crucial element for human life ... It is possible, why not" $(F 4,19)$ 
“...with parents, I can't discuss with them... In our society, everyone talks about all types of subjects, when it comes to sexuality and reproductive systems eh eh it is forbidden (shame)... This interview was a good experience for us and an opportunity to discuss this area which remains a taboo in our society" (M3, 18)

Knowledge about the concept of SRH is limited, and misinformation is widespread. Most participants have never heard of the term SRH and only know a few components like STIs and HIV/AIDS:

“... This is the first time that I hear the concept of sexual and reproductive health.... In high school, we studied HIV/AIDS..." (M12, 18)

However, the participants had a general understanding of this concept, which divided into sexuality and reproduction: "SRH is eh eh... a relationship with the reproductive system and sexuality..." (F5, 19)

A few participants linked SRH to marriage, mentioning that the area of sexuality and reproduction is only relevant to engage or married people:

"Sexuality is a taboo subject (shame) ... It is during the engagement period, or the preparation for marriage that one can discuss sexuality..." (F8, 18)

In addition, some participants linked sexual health to both sexes while reproductive health is limited to mother and newborn:

"Sexual health I think concerns women and men but reproductive health concerns mother and newborn" $(\mathrm{F} 11,18)$

The students also raised the notion of religion during the discussion:

“... The problem of the taboo (shameful) whereas according to our religion Islam, there is no shyness in religion... It is a question of social mentality and norms and has no relation with Islam" (M5, 18)

Concerning the importance of carrying out studies in the field of SRH, the participants unanimously insisted on the value of this type of research in society:

"Studies in this field are rare here in Morocco despite being very interesting" (M4, 22)

"... if the person wants to have good health concerning sexuality and reproduction, research and studies must be done on this topic ... Yes, it is very fascinating. Yes, it must be done" $(\mathrm{M} 7,21)$

\section{Discussion}

In any society, researching taboo topics is not easy. A taboo is a cultural or religious custom that prohibits people from talking about particular things that are offensive or shocking, for example those that refer to sex and bodily functions [11]. The harm affects individuals because of embarrassment or shame [12]. Our results highlighted that sexuality and reproduction are the most taboo subjects in our society. The young people raised the difficulty they find in talking about sexuality or reproduction with their parents or relatives despite their openness and willingness to do so. This result is corroborated by DeJong [13], who indicates that the older generation still holds the most outstanding authority despite the younger generation having an advantage in terms of education.

Regarding the knowledge of the concept of SRH, among the participants of both sexes, some linked SRH to human reproduction, contraception, and HIV/AIDS. This relates to the nature of the study population. These students had already studied human reproduction in high school, including, anatomy and physiology of the male and female reproductive systems, fertilization, fetal development, contraceptive techniques, STI prevention, and AIDS in their immunology courses [14]. On the other hand, there are those (mainly girls) who linked SRH to marriage which refers to social and religious norms that prohibit sex outside marriage. Indeed, and according to Rossier (2013), young women must include their sexuality in marriage: it is preferred that they should be virgins on the wedding day and faithful [15].

Regarding the importance of research in the area of SRH, the results were very favourable from both genders, who insisted on the value of this type of study to overcome the resulting taboo and to meet the needs of adolescents and young people to live their sexuality safely and satisfyingly [16].

\section{Conclusion}

This preliminary research demonstrated the acceptability of young students to discuss the topic of sexual and reproductive health. In addition, young people expressed their desire to be able to talk freely about sexuality and reproduction with their parents, teachers, and health professionals to overcome this taboo barrier that has long persisted in our society.

\section{Bibliography}

1. UNESCO, UNICEF, UNFPA, ONU Femmes, OMS, ONUSIDA, Principes directeurs internationaux sur l'éducation à la sexualité Une approche factuelle, (UNESCO, Paris, 2018)

2. UNPF, Programme of Action adopted at the International Conference on Population and Development Cairo, 5-13 September 1994, (UNPF, New York, 2014)

3. UNAIDS, Report on the global AIDS epidemic, (UNAIDS, Geneva, 2008) 
4. WHO, Programme d'orientation sur la santé des adolescents destiné aux prestataires de soins de santé, (WHO, Geneva, 2005)

5. Moroccan Ministry of Health, Enquête Nationale à Indicateurs Multiples et Santé des Jeunes ENIMSJ 2006-2007, (Ministère de la Santé du Maroc, Rabat, 2008)

6. UNFPA, Droits et accès universel à la santé sexuelle et reproductive, (UNFPA, Rabat, 2015)

7. C. Pope, N. Mays, BMJ, 311, 42-45 (1995)

8. E. Burke, F. Kébé, I. Flink, M. van Reeuwijk, A. leMay, Reprod Health Matter, 25:50, 43-54 (2017)

9. C.G. Loiselle, P. McGrath, D.F. Polit, Ch.T. Beck, Méthodes de recherche en sciences infirmières, approches quantitatives et qualitatives, (ERPI - Le Renouveau Pédagogique Editions, Québec, 2007)

10. L. Kohn, W. Christiaens, Reflets et Perspect. de la Vie Econ., 4(LIII), 67-82 (2014)

11. C. Gao, TPLS, 3(12), 2310-2314, (2013)

12. N.M. Landa, K. Fushai, Cogent Med, 5: 1501188 (2018)

13. J. DeJong, R. Jawad, I. M. Shepard, Reprod Health Matter,13(25), 49-59 (2005)

14. Centre D'études et de Recherches Demographiques (CERD), Santé de reproduction au Maroc: facteurs démographiques et socio-culturels, (CERD, Rabat,1998)

15. C.Rossier,N.Sawadogo,Population-F,68(1), 97-122 (2013)

16. C. Come Yélian Adohinzin, N.Meda, G.A. Ouédraogo, A.M.G. Belem, I.Sombié, A.Berthé, N.B.Kandala, G.D.Avimadjenon, L.Fond-Harmant, Santé Publique,28(4), 525-534(2016) 